\title{
Pregnancy-related acute kidney injury at high altitude: a retrospective observational study in a single center
}

\author{
Xin Li ${ }^{1}$, Xiaojing Wu', Muyin Zhang ${ }^{1}$, Lili Xu', Guohui $\mathrm{Li}^{2}$, Yumei Wen ${ }^{3}$ and Weiming Wang ${ }^{1 *}$
}

\begin{abstract}
Background: Pregnancy-related acute kidney injury (Pr-AKI) is associated with maternal and fetal morbidity and mortality. There are few studies focusing on Pr-AKI at high altitude in the literature.

Objectives: to investigate the incidence, etiology, clinical features and maternal-fetal outcomes of Pr-AKI in women living at high altitude.

Methods: 6,512 pregnant women attending the Department of Obstetrics \& Gynecology at local hospital from January 2015 to December 2018 were screened for Pr-AKI. Patients with serum creatinine above normal range(> $70 \mathrm{umol} / \mathrm{L}$ ) then underwent assessment to confirm the diagnosis of Pr-AKI. AKI was diagnosed and staged based on Kidney Disease Improving Global Outcomes(KDIGO) guideline. Individuals meeting the Pr-AKI criteria were recruited. Their clinical data were recorded and retrospectively analyzed.

Results: Pr-AKI was identified in 136/6512(2.09\%) patients. Hypertensive disorders of pregnancy(HDP) was the leading cause of Pr-AKI(35.3\%). 4(2.9\%) women died and the majority(86.1\%) had recovered renal function before discharge. Fetal outcomes were confirmed in 109 deliveries with gestational age $\geq 20$ weeks. Pre-term delivery occurred in 30(27.3\%) cases and perinatal deaths in 17(15.5\%). The rate of low birth weight infant(LBWI) and intrauterine growth restriction(IUGR) was 22.0 and $10.9 \%$ respectively. 16(14.5\%) infants were admitted to NICU after birth. Patients with HDP had a higher cesarean rate(56.3\%). More IUGR(25.0\%) and LBWI(37.8\%) were observed in their infants with a higher risk of admission to $\mathrm{NICU}(22.0 \%)$. High altitude might have an adverse impact on HDP-related Pr-AKI patients with earlier terminated pregnancy and more stillbirth/neonatal death. Logistic regression models indicated that uncontrolled blood pressure, high altitude and advanced AKI were associated with adverse fetal outcomes in HDP-related Pr-AKI patients.
\end{abstract}

Conclusions: Pr-AKI was not rare in high-altitude regions and caused severe fetal morbidities and mortalities. Uncontrolled blood pressure, high altitude and advanced AKI were all risk factors for adverse fetal outcomes in PrAKI patients, especially for those with hypertensive disorders of pregnancy.

Keywords: Acute kidney injury, Altitude, Hypertensive disorders of pregnancy, Pregnancy

\footnotetext{
* Correspondence: wwm11120@rihn.com.cn

'Department of Nephrology, Shanghai Ruijin Hospital, Shanghai Jiaotong

University School of Medicine, Shanghai, China

Full list of author information is available at the end of the article
}

(c) The Author(s). 2021 Open Access This article is licensed under a Creative Commons Attribution 4.0 International License, which permits use, sharing, adaptation, distribution and reproduction in any medium or format, as long as you give appropriate credit to the original author(s) and the source, provide a link to the Creative Commons licence, and indicate if changes were made. The images or other third party material in this article are included in the article's Creative Commons licence, unless indicated otherwise in a credit line to the material. If material is not included in the article's Creative Commons licence and your intended use is not permitted by statutory regulation or exceeds the permitted use, you will need to obtain permission directly from the copyright holder. To view a copy of this licence, visit http://creativecommons.org/licenses/by/4.0/ The Creative Commons Public Domain Dedication waiver (http://creativecommons.org/publicdomain/zero/1.0/) applies to the data made available in this article, unless otherwise stated in a credit line to the data. 


\section{Background}

Pregnancy-related acute kidney injury(Pr-AKI) is an important obstetric complication, featured by a rapid decline in renal function and a series of consequential clinical disorders, making it one of the leading causes of maternal and fetal morbidity and mortality. As a heterogeneous disease with various underlying etiologies, it encompasses a wide range of clinical manifestations of different severity and can occur at any time of the pregnancy. Not only is Pr-AKI strongly associated with adverse outcomes of both mothers and their offspring, but also has a profound impact on their future health by increasing the risks of developing hypertension, chronic kidney disease(CKD) and cardiovascular diseases later in their lives[1, 2].

Over the last few decades, there has been a dramatic decrease in the overall incidence of Pr-AKI worldwide, especially in developing countries, largely owning to the promotion of antenatal supervision and the improvement in maternal-infant care. However, in areas where adequate antenatal care and sanitary facilities are not available, pregnancy-related diseases still remain a huge public health problem. Etiology spectrum has also changed. Hypertensive diseases, especially preeclampsia and its related complications, have replaced traditional causes of Pr-AKI, such as septic abortion and hemorrhage, becoming the predominant etiologies of AKI in pregnant women[3-5]. For women living at high altitude, there is another challenge. As the arterial oxygen saturation begins to decrease with ascending altitude, the risk of arterial hypoxia and its associated complications increases and may have a negative effect on pregnancy. Previous studies have found that long exposure to hypoxia increases the incidence of preeclampsia and other obstetric complications, including intrauterine growth restriction(IUGR), stillbirth and neonatal mortality[6-8], but further investigations and detailed demonstrations are needed to support these observations.

The incidence of Pr-AKI reported in Chinese population ranged from 0.02 to $1.84 \%$ in recent years[3, 9], depending on different criteria they used to define AKI. However, most of the data came from tertiary hospitals in big cities. Data from undeveloped mountainous highlands was rare. Apart from social-economic factors, whether environmental factors play a role in the development of Pr-AKI and what influence they have on these patients deserve further discussion. Therefore, we conducted this research to investigate the incidence, etiology and clinical features of Pr-AKI at high altitude, and also looked into the risk factors of adverse pregnancy outcomes in these patients.

\section{Methods}

\section{Subjects}

A total of 6512 pregnant women were admitted to the Department of Obstetrics \& Gynecology, Diqing Tibetan
Autonomous Prefecture People's Hospital, Shangrila, Yunnan province from January 2015 to December 2018. The hospital is located on the eastern edge of QinghaiTibet plateau, a vast mountainous region with an average altitude of $3,300 \mathrm{~m}$ (ranging from $1,504 \mathrm{~m}$ to 5 , $545 \mathrm{~m}$ ) and 16 ethnic minorities. It serves as the only comprehensive medical institution in the region of Shangrila and receives patients from across this district as well as adjoining provinces including Tibet and Sichuan.

We used an electronic medical record system to screen for candidates meeting the following characteristics: (1) Pregnancy or puerperium; (2) Diagnoses relevant to renal involvement including renal failure/insufficiency, proteinuria, chronic kidney disease(CKD) and renal imaging abnormalities such as kidney stone and hydronephrosis; (3) high-risk pregnancy-related conditions, which according to the previous literature, are associated with an increased risk of AKI during pregnancy and puerperium, including hypertensive disorders of pregnancy, preeclampsia, eclampsia, HELLP syndrome, sepsis, hyperemesis gravidarum, ectopic pregnancy, antepartum/postpartum hemorrhage, amniotic fluid embolism, pyelonephritis, acute fatty liver of pregnancy, heart failure and multiple organ dysfunction syndrome; (4) According to the existing literature, serum creatini$\mathrm{ne}(\mathrm{Scr})>70 \mathrm{umol} / \mathrm{L}$ usually indicates renal dysfunction in pregnant women[10]. Therefore, Scr level $>70 \mu \mathrm{mol} / \mathrm{l}$ at least once during hospitalization was chosen as a threshold for the screening. After screening, all the candidates' electronic medical records were reviewed by a nephrologist and an obstetrician respectively to confirm a diagnosis of Pr-AKI. Candidates were required to provide antenatal supervision within 3 months or physical examination records within 12 months before admission in order to assess their baseline renal function. Those without records of baseline renal function or with preexisting CKD were excluded. Finally, 136 patients meeting the Pr-AKI criteria with detailed clinical data for analysis were recruited in this study. Patient recruitment was summarized in Fig. 1.

\section{Data collection}

Patients' demographic and clinical information were registered, including: (1) demographic data: age, ethnicity, residential altitude; (2) medical history: records of antenatal supervision, previous obstetric history, complications; (3) clinical information: blood pressure, body mass index, gestational age on admission, singleton/multiple births; (4) laboratory and imaging findings: blood cell count, urine test, liver enzyme, serum creatinine, albumin, uric acid, lipid, urinary ultrasound; (5) pregnancy outcomes: delivery mode, gestational age at delivery/ abortion, obstetric complications, maternal death. For 


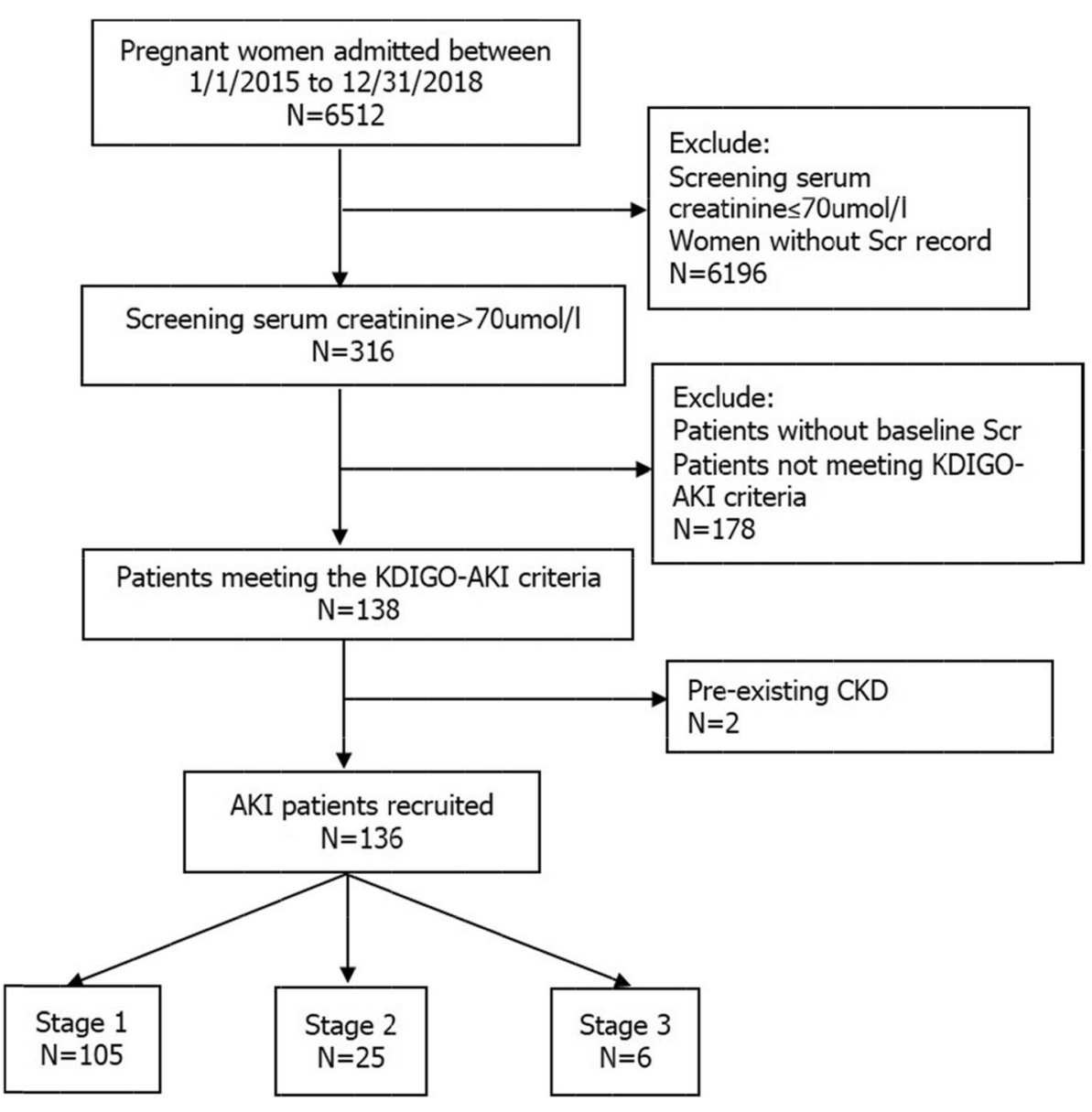

Fig. 1 Flow chat of patient recruitment

patients with gestational week $>20$ weeks, fetal outcomes were also recorded, including: preterm delivery, stillbirth/neonatal death, birth weight, Apgar score at $1 \mathrm{~min}$ after delivery, intrauterine growth restriction(IUGR), admission to neonatal intensive care unit(NICU), low birth weight infants(LBWI)). Baseline renal function for patients having regular antenatal supervision were obtained from the lowest serum creatinine value available from their supervision records. For patients without regular antenatal supervision, serum creatinine measurements at primary/secondary hospitals or from physical examination records within 12 months would be adopted as the baseline value. For those without any records of biochemical tests before hospitalization, the most recent serum creatinine prior to the occurrence of Pr-AKI would be adopted as the baseline value after assessment of our team. We analyzed the most likely causes of AKI according to assessment of all the available clinical and laboratory evidence. Consecutive Scr measurements during hospitalization were recorded until death, discharge or the patients were transferred to another hospital.

\section{Related definition}

Currently, there are no standardized criteria for the diagnosis of pregnancy-related AKI. Scr level typically is lower in pregnant women due to increased renal blood flow and glomerular hyperfiltration. Therefore, Scr level within normal range in non-pregnant women could reflect significant kidney injury in pregnancy. Criteria used in existing literature ranged from an increase in Scr to AKI requiring dialysis, among which the KDIGO guideline for AKI was the most widely used. In this study, we adopted the KDIGO guideline for Pr-AKI diagnosis and staging. According to the KDIGO guideline, an absolute increase in Scr level by $\geq 26.5 \mu \mathrm{mol} / \mathrm{l}(0.3 \mathrm{mg} / \mathrm{dl})$ within $48 \mathrm{~h}$ or a $50 \%$ increase from the baseline value within 7 days is considered as AKI[11]. AKI stage was evaluated according to the ratios of maximum Scr to baseline Scr with a ratio of $>$ $1.5,2$, and 3 denoting stages 1,2 , and 3 , respectively. A rise of $\mathrm{Scr} \geq 26 \mu \mathrm{mol} / \mathrm{l}$ within $48 \mathrm{~h}$ was considered as AKI stage 1 , and a maximum $\mathrm{Scr}>354 \mu \mathrm{mol} / \mathrm{l}$ was considered as AKI stage 3. Renal recovery was defined as final measured $\mathrm{Scr}<70 \mathrm{umol} / \mathrm{L}$ before discharge. 
The diagnosis of hypertensive disorders of pregnancy(HDP), preeclampsia, eclampsia was determined according to the definitions for hypertensive diseases from American College of Obstetricians and Gynecologists[12]. Diagnosis of HELLP syndrome, hyperemesis gravidarum, ectopic pregnancy, antepartum/postpartum hemorrhage, amniotic fluid embolism, pyelonephritis, acute fatty liver of pregnancy, heart failure and multiple organ dysfunction syndrome was made according to existing diagnostic criteria[13]. Term delivery was defined as delivery $\geq 37$ weeks' gestation. Preterm birth was defined as delivery $<37$ weeks' gestation. Stillbirth was defined as fetal death after 20 weeks' gestation. Neonatal death was defined when a newborn died before discharge from hospital. Low birth weight infant(LBWI) was defined as infants weighting less than 2500 gram. Intrauterine growth restriction(IUGR) was defined if birth weight of the newborn was below 10th percentile for given gestational age.

\section{Statistical analysis}

Normally distributed continuous data were summarized using means \pm standard deviation, and groups were compared using independent-sample t tests. Non-normally distributed data were summarized using medians(interquartile range) and compared across groups using Wilcoxon 2-sample tests. Categorical data were compared using Pearson $\chi^{2}$ test or Fisher exact test. Binary logistic regression model was used to explore the risk factors of adverse pregnancy outcomes. The statistical analysis was performed using SPSS version 22.0 for windows(SPSS Inc, Chicago, IL, USA). $\mathrm{P}<0.05$ was considered to be statistically significant.

\section{Results}

\section{Incidence and timing of Pr-AKI}

Pr-AKI was identified in 136/6512(2.09\%) pregnant women, approximately 1:48 pregnancies. 105(77.2\%) cases of Pr-AKI were stage 1 [median Scr $79 \mu \mathrm{mol} / \mathrm{L}(75-$ 88)]; $25(18.4 \%)$ cases were stage 2[median Scr $116 \mu \mathrm{mol} /$ $\mathrm{L}(103-125.5)$ ], and $6(4.4 \%)$ cases were stage 3[median Scr $319.5 \mu \mathrm{mol} / \mathrm{L}(173.8-438.8)]$. Their demographic and baseline clinical data were showed in Table 1.

Among 136 Pr-AKI patients, 24(17.6\%) cases occurred in the first trimester, $10(7.4 \%)$ in the second trimester, $91(66.9 \%)$ in the third trimester and $11(8.1 \%)$ during puerperium.

\section{Etiologies of Pr-AKI}

Hypertensive disorders of pregnancy(HDP) was the leading cause of Pr-AKI and was responsible for 48(35.3\%) cases, followed by sepsis(33 cases, 24.3\%) and hemorrhage(23 cases, $16.9 \%$ ).(Supplement Table 1).
Table 1 Demographic and baseline clinical information of 136 patients with Pr-AKI

\begin{tabular}{ll}
\hline Variables & Results(N= 136) \\
\hline Age (years) & $27.7 \pm 5.6$ \\
BMI $\left(\mathrm{kg} / \mathrm{m}^{2}\right)$ & $24.9 \pm 3.0$ \\
Residential altitude $(\mathrm{m})$ & $2804.4 \pm 628.3$ \\
Primipara/multipara & $76 / 60$ \\
Singleton/twin pregnancy & $133 / 3$ \\
SBP (mmHg) & $123.9 \pm 28.9$ \\
DBP (mmHg) & $80.6 \pm 20.1$ \\
Hemoglobin (g/L) & $116.4 \pm 28.7$ \\
Platelet count $\left(10^{9} / \mathrm{L}\right)$ & $182.5 \pm 91.2$ \\
ALT(IU/L) & $14(11-25)$ \\
AST(IU/L) & $20(16-29)$ \\
Serum ALB(g/L) & $29.4 \pm 7.8$ \\
SCr (umol/l) & $84(75-98)$ \\
Serum UA (umol/L) & $418.5 \pm 159.9$ \\
Total glyceride(mmol/L) & $3.8 \pm 2.3$ \\
Total cholesterol(mmol/L) & $5.6 \pm 2.1$ \\
\hline AKI a &
\end{tabular}

$A K I$ acute kidney injury, $B M I$ body mass index, $S B P$ systolic blood pressure; $D B P$ diastolic blood pressure; $A L B$ albumin; $S C r$ serum creatinine; UA uric acid; $A L T$ alanine transaminase, $A S T$ aspartate transaminase

In the first trimester, post-abortion $\operatorname{sepsis}(n=17)$ was the most common cause of Pr-AKI, followed by ectopic $\operatorname{pregnancy}(n=7)$. Hemorrhage due to placenta previa and abruption of placenta was responsible for 5 cases in the second trimester. Pr-AKI caused by ectopic pregnan$\operatorname{cy}(n=2)$ and $\operatorname{HDP}(n=2)$ was also noticed in this period. In the third trimester, HDP and its related diseases $(n=$ 46), especially preeclampsia $(n=33)$, became the most common causes of Pr-AKI, while antepartum/postpartum hemorrhage $(n=16)$ and obstetric infections $(n=8)$ took the second and third place respectively. During the puerperium, most Pr-AKI cases were caused by puerperal $\operatorname{sepsis}(n=9)$, and two cases were attributed to late postpartum hemorrhage due to retained placental tissues or dehiscence of cesarean section.

HELLP syndrome occurred in 3 patients with preeclampsia. 4 patients had acute fatty liver of pregnancy and 1 patient had amniotic fluid embolism. 3 cases were related to obstruction of urinary tract.

Besides the major cause, multiple factors could simultaneously contribute to the development of Pr-AKI as well. 49(36.0\%) patients had secondary or miscellaneous causes, including concomitant infections, insufficient renal perfusion and medication.

\section{Maternal and fetal outcomes of Pr-AKI}

There were $4(2.9 \%)$ cases of maternal death(2 patients with HELLP syndrome and 2 cases with rupture of uterus). No patients required emergency hemodialysis. 
Renal outcomes were confirmed in 115 patients ( 2 patients were transferred to other hospitals and 19 patients had no repeated Scr testing before discharge), and renal recovery occurred in $99(86.1 \%)$ patients. Patients with stage 1 AKI had the highest renal recovery rate compared with patients with stage 2 and $3 \operatorname{AKI}(93.1 \%$ vs. $68.2 \%$ vs. $50 \%, P=0.001$ ).

Fetal outcomes were confirmed in 109 deliveries with gestational age $\geq 20$ weeks, including two sets of twins. $42(38.9 \%)$ patients delivered via cesarean section and the mean gestational age at delivery was $37.1 \pm 3.3$ weeks. The average birth weight and Apgar score- $1 \mathrm{~min}$ after birth was $2935.2 \pm 589.3$ gram and $8.0 \pm 3.3$. Preterm delivery occurred in $30(27.3 \%)$ cases and perinatal deaths occurred in 17(15.5\%), including 8 stillbirths and 9 neonatal deaths. The rate of LBWI and IUGR was $22.0 \%(24$ cases $)$ and $10.9 \%(12$ cases $)$ respectively. $16(14.5 \%)$ infants were admitted to NICU after birth due to prematurity-related complications.

\section{Pr-AKI in patients with hypertensive disorders of pregnancy}

48 cases(including 2 twin pregnancies) of $\mathrm{Pr}$-AKI were related to $\operatorname{HDP}(2$ in the 2nd and 46 in the 3rd trimester), including 35 cases of preeclampsia, 2 cases of eclampsia and 3 cases of HELLP syndrome. Compared with 50 non-HDP patients(including 1 twin pregnancy), HDP-related Pr-AKI patients had a lower level of serum albumin $(24.9 \pm 5.6$ vs. $27.5 \pm 5.3, P=0.02)$ and a higher level of uric $\operatorname{acid}(477.1 \pm 82.8$ vs. $406.2 \pm 115.7, P=0.01)$. Cesarean rate was much higher in HDP-related Pr-AKI patients than their counterparts $(56.3 \%$ vs. $31.3 \%, P=$ 0.014). As for pregnancy outcomes, infants delivered by patients with HDP were more likely to have $\operatorname{IUGR}(25.0 \%$ vs. $2.3 \%, \quad P=0.003) \quad$ or low birth weight( $37.8 \%$ vs. $15.9 \%, P=0.02)$, and also at higher risk of admission to $\mathrm{NICU}(22.0 \%$ vs. $6.5 \%, P=$ 0.019).(Table 2).

\section{Impact of altitude on Pr-AKI patients}

In order to assess the impact of altitude on patients with Pr-AKI, they were further divided into two groups according to their residential altitude: the low-altitude group(residential altitude $<3000 \mathrm{~m}$ ) and the high-altitude group(residential altitude $\geq 3000 \mathrm{~m}$ ).

There were no statistically significant differences between the two groups in either clinical features or pregnancy outcomes(Supplement Table 2). However, in the

Table 2 Comparison of clinical features and pregnancy outcomes between HDP-related and non-HDP Pr-AKI patients

\begin{tabular}{|c|c|c|c|}
\hline Variables & $\begin{array}{l}\text { HDP-related } \\
N=48 \text { (50 fetuses) }\end{array}$ & $\begin{array}{l}\text { Non-HDP } \\
N=50 \text { (50 fetuses) }\end{array}$ & $P$ value \\
\hline Age (years) & $28.3 \pm 5.1$ & $26.9 \pm 6.0$ & 0.255 \\
\hline $\mathrm{SBP}(\mathrm{mmHg})$ & $154.3 \pm 19.0$ & $110.6 \pm 13.5$ & $<0.001$ \\
\hline $\mathrm{DBP}(\mathrm{mmHg})$ & $101.4 \pm 15.3$ & $69.3 \pm 10.1$ & $<0.001$ \\
\hline Serum ALB (g/L) & $24.9 \pm 5.6$ & $27.5 \pm 5.3$ & 0.020 \\
\hline $\mathrm{SCr}(\mathrm{umol} / \mathrm{L})$ & $86(77-98)$ & $90(78-111)$ & $0.355^{\mathrm{a}}$ \\
\hline Serum UA (umol/L) & $477.1 \pm 82.8$ & $406.2 \pm 115.7$ & 0.001 \\
\hline Maternal death (n\%) & $2(4.0 \%)$ & $2(3.7 \%)$ & $1.000^{\mathrm{b}}$ \\
\hline $\begin{array}{l}\text { Gestational age at delivery } \\
\text { (in weeks) }\end{array}$ & $37.1 \pm 2.8$ & $37.0 \pm 4.0$ & 0.858 \\
\hline Delivery via cesarean section (n\%) & $27(56.3 \%)$ & $15(31.3 \%)$ & 0.014 \\
\hline Pre-term birth (n\%) & $17(35.4 \%)$ & $12(26.1 \%)$ & 0.328 \\
\hline Stillbirth/neonatal death (n\%) & $6(13.6 \%)$ & $10(21.3 \%)$ & 0.339 \\
\hline Neonatal weight (g) & $2770.2 \pm 647.4$ & $3023.8 \pm 523.7$ & 0.054 \\
\hline Apgar score at 1 min & $7.8 \pm 3.3$ & $7.7 \pm 3.7$ & 0.923 \\
\hline IUGR (n\%) & $11(25.0 \%)$ & $1(2.3 \%)$ & $0.003^{b}$ \\
\hline Admission to NICU (n\%) & $11(22.0 \%)$ & $3(6.5 \%)$ & $0.019^{b}$ \\
\hline LBWI (n\%) & $17(37.8 \%)$ & $7(15.9 \%)$ & 0.020 \\
\hline Renal recovery on discharge (n\%) & $29(76.3 \%)$ & $35(85.4 \%)$ & 0.305 \\
\hline
\end{tabular}

$A K I$ acute kidney injury; HDP hypertensive disorders of pregnancy; SBP systolic blood pressure; $D B P$ diastolic blood pressure; $A L B$ albumin; SCr serum creatinine; UA uric acid; IUGR intrauterine growth restriction; LBWI low birth weight infant; NICU neonatal intensive care unit

Values for categorical variables were given as number (percentage); values for continuous variables were given as mean \pm standard deviation or median (interquartile range)

$P<0.05$ was considered to be statistically significant

a: Wilcoxon 2-sample tests

${ }^{\mathrm{b}}$ : Fisher exact test 
HDP-related Pr-AKI subgroup, those living at high altitude had earlier terminated pregnancy $(36.3 \pm 3.1$ weeks vs. $38.0 \pm 1.8$ weeks, $P=0.03$ ) and more stillbirth/neonatal deaths $(24.0 \%$ vs. $0 \%, P=0.029)$ than their counterparts. Apgar-1 min scores(7.0 \pm 3.8 vs. $8.8 \pm 2.1, P=0.091)$ and birth weight $(2625.0 \pm 694.4 \mathrm{~g}$ vs. $2963.9 \pm 537.6 \mathrm{~g}, P=$ $0.093)$ were also lower compared with patients from low altitude in this subgroup.(Table 3).

\section{Risk factors for adverse pregnancy outcomes}

We used binary logistic regression models to explore potential risk factors for adverse pregnancy outcomes of Pr-AKI patients. Variables retained in the model were maternal age, residential altitude, AKI staging, and the highest systolic blood pressure after admission.

Among patients without HDP, only AKI staging was found associated with higher risk of stillbirth/neonatal death[OR 4.0(95 \%CI 1.34,11.99), $P=0.013$ ].
Among patients with HDP, high blood pressure was the strongest risk factor related to adverse fetal outcomes. For every $10 \mathrm{mmHg}$ increase in systolic blood pressure, the risk of preterm birth, LBWI, admission to NICU and IUGR would increase by 2.55 times[95 \%CI $(1.24,5.21), P=$ 0.011], 2.16 times[95 \%CI(1.18,3.95), $P=0.013], 2.02$ times[95 \%CI $(1.11,3.66), P=0.21]$ and 1.75 times[95 \%CI (1.03,2.99), $P=0.39$ ] respectively. High altitude was found associated with elevated risk of LBWI[OR 7.30, $95 \% \mathrm{CI}(1.12,47.38), P=0.037]$. AKI staging was associated with higher risk of preterm birth[OR 17.6, $95 \% \mathrm{CI}(1.95$, 158.5), $P=0.011$ ] and stillbirth/neonatal death[OR 11.5, $95 \% \mathrm{CI}(1.15,114.9), P=0.038]$. (Table 4).

\section{Comments}

The landscape of pregnancy-related AKI has been changing dramatically over the last few decades. There has been a sharp decline in the overall incidence of Pr-AKI, not just in the developed world but also in the low-

Table 3 Subgroup analysis of clinical features and pregnancy outcomes between Pr-AKI women living at low and high altitudes

\begin{tabular}{|c|c|c|c|c|c|c|}
\hline \multirow[t]{2}{*}{ Variables } & \multicolumn{3}{|c|}{ Without HDP $(N=50)$} & \multicolumn{3}{|c|}{ With HDP $(N=48)$} \\
\hline & $\begin{array}{l}\mathrm{LA} \\
N=28\end{array}$ & $\begin{array}{l}\mathrm{HA} \\
N=22\end{array}$ & $P$ value & $\begin{array}{l}\mathrm{LA} \\
N=21\end{array}$ & $\begin{array}{l}\mathrm{HA} \\
N=27\end{array}$ & $P$ value \\
\hline Residential altitude (m) & $2207.5 \pm 345.4$ & $3415.2 \pm 244.4$ & - & $2344.8 \pm 199.7$ & $3336.3 \pm 146.9$ & - \\
\hline Age (years) & $27.6 \pm 6.7$ & $26.0 \pm 4.6$ & 0.333 & $27.9 \pm 5.4$ & $28.5 \pm 5.0$ & 0.686 \\
\hline BMI $\left(\mathrm{kg} / \mathrm{m}^{2}\right)$ & $24.6 \pm 2.4$ & $24.2 \pm 2.6$ & 0.683 & $27.3 \pm 5.2$ & $27.3 \pm 3.8$ & 0.983 \\
\hline $\mathrm{SBP}(\mathrm{mmHg})$ & $111.0 \pm 14.9$ & $110.6 \pm 11.4$ & 0.915 & $155.5 \pm 18.2$ & $153.8 \pm 19.3$ & 0.751 \\
\hline $\mathrm{DBP}(\mathrm{mmHg})$ & $68.7 \pm 10.2$ & $69.6 \pm 10.3$ & 0.736 & $103.3 \pm 13.6$ & $101.5 \pm 17.7$ & 0.687 \\
\hline Hemoglobin (g/L) & $105.4 \pm 24.1$ & $108.7 \pm 38.6$ & 0.718 & $125.5 \pm 22.8$ & $115.0 \pm 29.9$ & 0.187 \\
\hline Serum ALB (g/L) & $27.4 \pm 5.7$ & $27.8 \pm 4.7$ & 0.826 & $25.7 \pm 5.4$ & $23.6 \pm 6.0$ & 0.212 \\
\hline SCr (umol/L) & $91(76-109)$ & $90(80-113)$ & $0.872^{a}$ & $88(76-97)$ & $86(77-100)$ & $0.592^{\mathrm{a}}$ \\
\hline Serum UA (umol/L) & $429.1 \pm 276.5$ & $428.2 \pm 112.5$ & 0.989 & $481.4 \pm 67.9$ & $475.7 \pm 91.5$ & 0.809 \\
\hline Maternal death (n\%) & $1(3.4 \%)$ & $1(4.5 \%)$ & $1.000^{b}$ & $1(4.7 \%)$ & $1(3.7 \%)$ & $0.511^{b}$ \\
\hline $\begin{array}{l}\text { Gestational age at delivery } \\
\text { (in weeks) }\end{array}$ & $36.9 \pm 3.4$ & $37.0 \pm 4.6$ & 0.890 & $38.0 \pm 1.8$ & $36.3 \pm 3.1$ & 0.030 \\
\hline Delivery by cesarean section (n\%) & $8(30.8 \%)$ & $7(31.8 \%)$ & 0.938 & $10(47.6 \%)$ & $17(63.0 \%)$ & 0.288 \\
\hline Pre-term birth (n\%) & $7(26.9 \%)$ & $5(25.0 \%)$ & 0.883 & $5(23.8 \%)$ & $12(44.4 \%)$ & 0.138 \\
\hline Stillbirth/neonatal death (n\%) & $6(23.1 \%)$ & $4(19.0 \%)$ & $1.000^{\mathrm{b}}$ & 0 & $6(24.0 \%)$ & $0.029^{b}$ \\
\hline Neonatal weight (g) & $3018.2 \pm 580.1$ & $3030.6 \pm 461.8$ & 0.942 & $2963.9 \pm 537.6$ & $2625.0 \pm 694.4$ & 0.093 \\
\hline Apgar score at $1 \mathrm{~min}$ & $7.6 \pm 3.8$ & $7.8 \pm 3.7$ & 0.904 & $8.8 \pm 2.1$ & $7.0 \pm 3.8$ & 0.091 \\
\hline IUGR (n\%) & 0 & $1(5.3 \%)$ & $0.442^{b}$ & $3(20.0 \%)$ & $8(27.6 \%)$ & $0.480^{b}$ \\
\hline Admission to NICU (n\%) & $2(7.7 \%)$ & $1(5.0 \%)$ & $1.000^{\mathrm{b}}$ & $5(27.8 \%)$ & $6(24.0 \%)$ & $1.000^{\mathrm{b}}$ \\
\hline LBWI (n\%) & $5(20.8 \%)$ & $2(10.0 \%)$ & $0.428^{b}$ & $5(25.0 \%)$ & $12(48.0 \%)$ & 0.114 \\
\hline Renal recovery on discharge (n\%) & $22(91.7 \%)$ & $13(76.5 \%)$ & $0.212^{b}$ & $9(64.3 \%)$ & $20(83.3 \%)$ & $0.245^{b}$ \\
\hline
\end{tabular}

$A K I$ acute kidney injury; HDP hypertensive disorders of pregnancy; $L A$ low altitude ( $<2500 \mathrm{~m}$ ); HA high altitude ( $\geq 2500 \mathrm{~m}$ ); $B M I$ body mass index; $S B P$ systolic blood pressure; $D B P$ diastolic blood pressure; $A L B$ albumin; $S C r$ serum creatinine; UA uric acid; IUGR intrauterine growth restriction; $L B W I$ low birth weight infant; NICU neonatal intensive care unit

Values for categorical variables were given as number (percentage); values for continuous variables were given as mean \pm standard deviation or median (interquartile range)

$P<0.05$ was considered to be statistically significant

a: Wilcoxon 2-sample tests

b. Fisher exact test 
Table 4 Binary logistic regression models for potential risk factors of adverse pregnancy outcomes in Pr-AKI patients with and without HDP

\begin{tabular}{|c|c|c|c|c|c|}
\hline \multicolumn{6}{|l|}{ Without HDP } \\
\hline \multirow[t]{2}{*}{ Variable } & \multicolumn{5}{|c|}{ OR (95 \%Cl) } \\
\hline & $\begin{array}{l}\text { Preterm } \\
\text { birth }\end{array}$ & $\begin{array}{l}\text { Stillbirth/ } \\
\text { neonatal } \\
\text { death }\end{array}$ & LBWI & $\mathrm{NICU}$ & IUGR \\
\hline $\begin{array}{l}\text { Maternal age } \\
\text { (1 year) }\end{array}$ & $\begin{array}{l}1.10(0.97, \\
1.26)\end{array}$ & $1.12(0.97,1.29)$ & $\begin{array}{l}1.02(0.89, \\
1.18)\end{array}$ & $\begin{array}{l}0.95(0.76 \\
1.19)\end{array}$ & NA \\
\hline $\begin{array}{l}\text { SBPmax } \\
\text { (10mmhg) }\end{array}$ & $\begin{array}{l}0.69(0.41 \\
1.16)\end{array}$ & $0.71(0.40,1.26)$ & $\begin{array}{l}1.29(0.66 \\
2.53)\end{array}$ & $\begin{array}{l}1.25(0.42 \\
3.24)\end{array}$ & NA \\
\hline High altitude & $\begin{array}{l}1.06(0.24 \\
4.74)\end{array}$ & $0.72(0.13,4.00)$ & $\begin{array}{l}0.39(0.06 \\
2.59)\end{array}$ & $\begin{array}{l}0.60(0.05 \\
7.39)\end{array}$ & NA \\
\hline AKI staging & $\begin{array}{l}2.67(0.93 \\
7.67)\end{array}$ & $\begin{array}{l}4.00(1.34 \\
11.99)\end{array}$ & $\begin{array}{l}2.55(0.74 \\
8.73)\end{array}$ & $\begin{array}{l}0.87(0.11 \\
6.88)\end{array}$ & NA \\
\hline \multicolumn{6}{|l|}{ With HDP } \\
\hline \multirow[t]{2}{*}{ Variable } & \multicolumn{5}{|c|}{ OR (95 \%Cl) } \\
\hline & $\begin{array}{l}\text { Preterm } \\
\text { birth }\end{array}$ & $\begin{array}{l}\text { Stillbirth/ } \\
\text { neonatal } \\
\text { death }\end{array}$ & LBWI & $\mathrm{NICU}$ & IUGR \\
\hline $\begin{array}{l}\text { Maternal age } \\
\text { (1 year) }\end{array}$ & $\begin{array}{l}0.93(0.78 \\
1.12)\end{array}$ & $0.95(0.71,1.27)$ & $\begin{array}{l}0.91(0.76 \\
1.08)\end{array}$ & $\begin{array}{l}0.92(0.77 \\
1.10)\end{array}$ & $\begin{array}{l}0.86(0.70 \\
1.06)\end{array}$ \\
\hline $\begin{array}{l}\text { SBPmax } \\
\text { (10mmhg) }\end{array}$ & $\begin{array}{l}2.55(1.24 \\
5.21)\end{array}$ & $0.87(0.39,1.93)$ & $\begin{array}{l}2.16(1.18 \\
3.95)\end{array}$ & $\begin{array}{l}2.02(1.11 \\
3.66)\end{array}$ & $\begin{array}{l}1.75(1.03, \\
2.99)\end{array}$ \\
\hline $\begin{array}{l}\text { High } \\
\text { altitude(> } \\
3000 \text { m) }\end{array}$ & $\begin{array}{l}9.08(0.98 \\
84.4)\end{array}$ & NA & $\begin{array}{l}7.30(1.12 \\
47.38)\end{array}$ & $\begin{array}{l}1.21(0.24 \\
6.13)\end{array}$ & $\begin{array}{l}2.88(0.49 \\
16.64)\end{array}$ \\
\hline AKI staging & $\begin{array}{l}\text { 17.6(1.95, } \\
158.5)\end{array}$ & $\begin{array}{l}11.5(1.15 \\
114.9)\end{array}$ & $\begin{array}{l}5.06(0.65 \\
39.39)\end{array}$ & $\begin{array}{l}0.80(0.15 \\
4.19)\end{array}$ & $\begin{array}{l}3.98(0.68 \\
23.21)\end{array}$ \\
\hline
\end{tabular}

$A K I$ acute kidney injury; HDP hypertensive disorders of pregnancy; SBPmax the highest systolic blood pressure after admission; $L B W I$ low birth weight infant;

IUGR intrauterine growth restriction; NICU neonatal intensive care unit; NA not applicable

$P<0.05$ was considered to be statistically significant

middle-income countries, mostly due to improvement in antenatal care and a significant decrease of septic abortion. The incidence of $\mathrm{Pr}-\mathrm{AKI}$ in developed countries has remarkably reduced from approximately $1 / 3000$ to $1 / 15,000-1 / 20,000$ pregnancies from the $1960 \mathrm{~s}$ to the end of last century[14]. In developing countries such as India and China, the overall condition is also greatly improving. India has revealed a considerable decline in the proportion of Pr-AKI among hospitalized patients from $15.2 \%$ in the $1980 \mathrm{~s}$ to $1.5-4.6 \%$ in the $2010 \mathrm{~s}[15,16]$. In the meantime, maternal mortality has reduced to $5.79 \%$ from initial high value $20 \%$ in the $1980 \mathrm{~s}$, and the progression of Pr-AKI to ESRD decreased to $1.4 \%$ from $6.15 \%[15]$.

As a developing country and also the world's most populous nation, China has made impressive progress in fighting against severe obstetric diseases and reducing maternal-neonatal mortalities. The maternal mortality ratios have declined fast from $108.7 / 100,000$ to $21.8 / 100$, 000 livebirths from 1996 to 2015[17], and the infant mortality has also decreased from $46.7 \%$ o to $8.1 \%$ from 1992 to 2015[18]. In recent years, reported incidence of Pr-AKI in China is ranging from 0.02 to $1.84 \%[3,9]$, along with declining maternal and neonatal mortalities. Despite these encouraging achievements, huge regional discrepancies still exist within the country. In less economically developed western provinces, pregnancyrelated diseases including Pr-AKI remain a critical public health problem to local people due to limited medical resources. According to the 2010 population census of China[19, 20], $58.6 \%$ of the population in western China live in rural areas (eastern China: $40 \%$ ), $34.2 \%$ of them belong to ethnic minority groups (eastern China: $2.1 \%$ ) and $10.1 \%$ of women are illiterate (eastern China: $4.7 \%$ ). Underdevelopment, ethnic diversity, and geographical conditions all present great challenges to ensuring universal access to medical resources. In our study, the incidence of Pr-AKI in the region of Shangrila was $2.09 \%$, higher than previous data reported by other Chinese authors. Given that we had excluded patients who delivered out of hospital as well as those unable to provide baseline renal function, this figure was likely to be by far underestimated.

There is also an urgent need to raise the awareness of Pr-AKI among medical practitioners in local medical institutions. Pr-AKI is not rare among women of childbearing age, and the incidence can be extremely high in patients with particular obstetric complications. It affects approximately $5-17 \%$ of patients with severe preeclampsia $[4,9,21]$ and $7-60 \%$ of patients with HELLP syndrome[9, 22, 23]. However, a recent study revealed that only $4.0 \%$ of AKI events were initially diagnosed on the discharge record[24]. Patients at early stage of renal impairment sometimes present with Scr levels within normal range due to physiological glomerular hyperfiltration. In our study, the median Scr level was 84umol/ l(0.95 mg/dl). Only 24(17.6\%) patients had a Scr level > $105 \mathrm{umol} / \mathrm{l}(1.1 \mathrm{mg} / \mathrm{dl})$. In the setting of pregnancy, any Scr value above $70.72 \mathrm{umol} / \mathrm{l}(0.8 \mathrm{mg} / \mathrm{dl})$ should be considered abnormality. That's why we used 70umol/1 as a cut-off value for screening Pr-AKI candidates in this study. The relative change of Scr within a time period should also be taken into account, rather than just using a fixed upper limit value when making an AKI diagnosis. Judging by Scr levels alone will miss mild AKI events in patients with low baseline Scr, leading to underestimation in clinical practice.

In general, maternal outcome in less severe $\mathrm{Pr}$-AKI is favorable. However, severe Pr-AKI is associated with significantly increased mortality, especially among those requiring dialysis. Recent studies from China and India report that maternal mortality rate associated with severe $\mathrm{Pr}$-AKI ranges from 4.0 to $8 \%[3,17,25]$. In the long run, approximately 4-9\% of women with severe Pr- 
AKI requiring dialysis will remain dialysis-dependent for another 4-6 months after delivery[26]. The rate of progression to end-stage renal disease from Pr-AKI ranges from 1.4 to $2.5 \%[17,27]$.

Pr-AKI also presents a harsh challenge for the newborns. Perinatal mortality including intrauterine death, stillbirth and neonatal death occurs in $23.5-54 \%$ of Pr-AKI patients $[5,25]$, and the odds of perinatal mortality increase 3.39 fold when compared with pregnancies without Pr-AKI[27]. Pr-AKI is also linked to preterm delivery and disturbance of fetal development, resulting in IUGR, low birth weight or infant small for gestational age. This trend is particularly significant in patients suffering from HDP or preeclampsia. Prematurity and small babies are not only associated with prenatal death and fetal distress, but also have potential long-term detrimental impacts. Preterm is a strong risk factor for development of CKD in adulthood because of the delayed and insufficient kidney growth which may result in a low nephron number[28]. Additionally, it has been demonstrated that CKD, small and preterm babies will be at high risk for development of diabetes, metabolic syndrome and cardiovascular diseases in adulthood[29, 30].

We also looked into the impact of high altitude on pregnant women, especially those with HDP. Previous literature has reported a correlation between long exposure to high altitude and adverse fetal outcomes. The odds of stillbirth and preterm increase by 3.9fold and 1.7-fold respectively at high altitude relative to low altitude[31]. High altitude is also associated with lower birth weight, reducing fetal birth weight by an average of $102 \mathrm{~g}$ for every $1000 \mathrm{~m}$ increase in elevation[32]. These findings can be partially explained by maternal arterial hypoxia from high altitude exposure. In addition to this, long exposure to high altitude can interfere with effective transportation of oxygenated blood to the utero-placental circulation. High altitude decreases the pregnancy-induced increase in cardiac output and blood volume, likely due to a failure to reduce peripheral vascular resistance[33]. It also interferes with maternal vascular adjustment to pregnancy, such as the growth and remodeling of the uterine artery and other uteroplacental vessels, attenuating the normal increase of uterine artery diameter and blood flow[34]. It also alters patterns of blood flow redistribution, for example, by reducing the proportion of common iliac blood flow distributed to the uterine versus the external iliac artery, resulting in lower uterine artery blood flow near term $[34,35]$. In the presence of preeclampsia, which is commonly accompanied by uteroplacental vascular dysfunction and placental ischemia, insufficient maternal arterial oxygenation and compromised compensatory mechanism from long exposure to high altitude will further aggravate placental insufficiency and fetal intrauterine hypoxia. Moreover, long exposure to high altitude is also responsible for the pathogenesis and development of preeclampsia. The incidence of preeclampsia increases by approximately 3 -fold at high altitude[36]. Hypoxia regulates the production of numerous vascular endothelial regulatory factors known to influence vascular function during pregnancy and/or to be associated with preeclampsia, including endothelin-1, thromboxane A2, prostacyclin and tumor necrosis factor alpha[37]. Animal experiments also confirm that long-term high altitude hypoxia during gestation suppresses large conductance $\mathrm{Ca} 2+$-activated $\mathrm{K}+(\mathrm{BKCa})$ channel function in uterine arteries, which is of critical importance in pregnancymediated increase in uterine artery vasodilation and blood flow, resulting in the dysfunction of uterine placental circulation and the development of preeclampsia[38]. Hypoxia can also activate placental renin-angiotensin system, leading to placental dysfunction and oxidative stress response, which enhance the vasoconstrictor effect of locally-generated angiotensin II and contribute to the restricted fetal growth characteristic of preeclampsia[39, 40]. As preeclampsia has proved to be an important contributory factor to adverse fetal outcomes, we believe that the interaction between hypoxia and preeclampsia may have synergistic effects and enhance the adverse impacts of preeclampsia on pregnancy outcomes.

\section{Strengths and limitations}

Clinical researches focusing on AKI within pregnancy, especially for women from high altitude are rare, and the association between environmental factors including altitude and pregnancy outcomes in Pr-AKI patients is scarcely discussed in the literature. In this study, we made a comprehensive analysis of the clinical data of Pr-AKI patients in the high-altitude regions of western China and also looked into the risk factors of adverse pregnancy outcomes in these people. We found that uncontrolled blood pressure, high altitude and advanced AKI were all risk factors for adverse fetal outcomes in HDP-related Pr-AKI patients, while in Pr-AKI patients without HDP, only AKI stage was found associated with worse fetal outcomes. Our finding emphasized the importance of blood pressure control among hypertensive pregnant women, as we revealed a strong connection between uncontrolled blood pressure and almost all types of adverse outcomes in their fetus. There might also be a link between high altitude and adverse fetal outcomes including stillbirth/neonatal death and LBWI 
especially in HDP-related Pr-AKI patients. It highlighted the necessity of intensive perinatal care and monitoring for patients from high altitude in the presence of HDP and advanced AKI stages, and called for more reasonable distribution of social and medical resources to patients at high risk in areas with limited resources. With more than 140 million people living at high altitude all around the world and the high prevalence of HDP among pregnant women in less economically developed regions, this finding was of great clinical and social-economic significance.

Our study has some limitations. Firstly, histological data were unavailable because renal biopsy couldn't be performed due to technological limitations of the local hospital. Etiologies of Pr-AKI that relied on renal biopsy might be misdiagnosed or get overlapped with preexisting underlying renal diseases which were commonly seen in women of childbearing age, for example, IgA nephropathy and lupus nephritis. The second limitation is the incomplete follow-up records. Pregnancy outcomes of critically ill patients who had to be transferred to tertiary hospitals located at lower altitudes were not included in our analysis. Besides, most patients lost to follow-up after discharge, making it difficult to assess their long-term renal outcomes. Thirdly, confounding factors that might be related to pregnancy outcomes such as nutritional status, income and education levels were not analyzed in this study due to the limitation of data sources and small sample size. Finally, since our data came from a single-center study with a small group of patients, studies with enlarged sample size or even multi-center prospective cohorts were required to validate our findings.

\section{Conclusions}

In summary, this study revealed that Pr-AKI was not rare in high-altitude regions. It affected around $2.09 \%$ of the pregnant women but this figure might be by far underestimated. Although the overall maternal outcome was favorable, it still caused severe fetal morbidities and mortalities. More attention should be paid to patients with HDP, because it was not only the main cause of PrAKI but also led to worse pregnancy outcomes compared with patients with other etiologies. Uncontrolled blood pressure, high altitude, and advanced AKI were all risk factors for adverse fetal outcomes in HDP-related Pr-AKI patients. Thus more intensive and timely perinatal care and monitoring were needed for high-risk patients in order to improve their prognosis.

\section{Abbreviations}

Pr-AKI: Pregnancy-related acute kidney injury; KDIGO: Kidney Disease Improving Global Outcomes; HDP: Hypertensive disorders of pregnancy; BMI: Body mass index; SBP: Systolic blood pressure; DBP: Diastolic blood pressure; ALB: Albumin; SCr: Serum creatinine; UA: Uric acid; ALT: Alanine transaminase; AST: Aspartate transaminase; IUGR: Intrauterine growth restriction; LBWI: Low birth weight infant; NICU: Neonatal intensive care unit

\section{Supplementary Information}

The online version contains supplementary material available at https://doi. org/10.1186/s12882-021-02418-7.

Additional file 1: Etiologies of Pr-AKI in different phases of pregnancy

Additional file 2: Comparison of clinical features and pregnancy outcomes between Pr-AKI patients from low and high altitude

Additional file 2: Comparison of clinical features and pregnancy outcomes between Pr-AKI patients from low and high altitude

\section{Acknowledgements}

Not applicable.

\section{Authors' contributions}

$\mathrm{XL}$ conceived of the study, oversaw data analyses and drafted the manuscript. XW analyzed the data and drafted parts of the manuscript. MZ, $G L$ and YW wrote parts of the manuscript and contributed to interpretation of the data. WW approved the article and worked as the correspondence author of this study. All authors have read and approved the final manuscript.

Funding

This study was funded by National Natural Science Foundation of China(NSFC)(81870492).

Availability of data and materials

The datasets generated and analyzed during the current study are available from the corresponding author on reasonable request.

\section{Declarations}

Ethics approval and consent to participate

This retrospective study was approved by the ethics committee of Shanghai Ruijin Hospital, School of Medicine, Shanghai Jiaotong University. The ethics committee of hospital waived the need for written informed consent from the patients.

Consent for publication

Not applicable.

Competing interests

The authors declare that they have no competing interests.

\section{Author details}

${ }^{1}$ Department of Nephrology, Shanghai Ruijin Hospital, Shanghai Jiaotong University School of Medicine, Shanghai, China. ${ }^{2}$ Department of Internal Medicine, Diqing Tibetan Autonomous Prefecture People's Hospital, Shangrila, China. ${ }^{3}$ Department of Obstetrics and Gynecology, Diqing Tibetan Autonomous Prefecture People's Hospital, Shangrila, China.

Received: 23 September 2020 Accepted: 25 May 2021

Published online: 09 June 2021

\section{References}

1. Silver SA, Siew ED. Follow-up Care in Acute Kidney Injury: Lost in Transition. Adv Chronic Kidney Dis. 2017;24(4):246-52.

2. Cunningham MW Jr, LaMarca B. Risk of cardiovascular disease, end-stage renal disease, and stroke in postpartum women and their fetuses after a hypertensive pregnancy. Am J Physiol Regul Integr Comp Physiol. 2018; 315(3):R521-r528.

3. Liu YM, Bao HD, Jiang ZZ, Huang YJ, Wang NS. Pregnancy-related Acute Kidney Injury and a Review of the Literature in China. Intern Med. 2015; 54(14):1695-703.

4. Cooke WR, Hemmilä UK, Craik AL, Mandula CJ, Mvula P, Msusa A, Dreyer G, Evans R. Incidence, aetiology and outcomes of obstetric-related acute 
kidney injury in Malawi: a prospective observational study. BMC Nephrol. 2018;19(1):25.

5. Prakash J, Ganiger VC, Prakash S, lqbal M, Kar DP, Singh U, Verma A. Acute kidney injury in pregnancy with special reference to pregnancy-specific disorders: a hospital based study (2014-2016). J Nephrol. 2018;31(1):79-85.

6. Julian CG. High altitude during pregnancy. Clin Chest Med. 2011;32(1): $21-31$.

7. Bailey B, Euser AG, Bol KA, Julian CG, Moore LG. High-altitude residence alters blood-pressure course and increases hypertensive disorders of pregnancy. J Matern Fetal Neonatal Med 2020:1-8.

8. Keyes LE, Armaza JF, Niermeyer S, Vargas E, Young DA, Moore LG. Intrauterine growth restriction, preeclampsia, and intrauterine mortality at high altitude in Bolivia. Pediatr Res. 2003;54(1):20-5.

9. Huang $C$, Chen S. Acute kidney injury during pregnancy and puerperium: a retrospective study in a single center. BMC Nephrol. 2017;18(1):146.

10. Krane NK, Hamrahian M. Pregnancy: kidney diseases and hypertension. Am J Kidney Dis. 2007:49(2):336-45.

11. Kellum JA, Lameire N. Diagnosis, evaluation, and management of acute kidney injury: a KDIGO summary (Part 1). Crit Care. 2013;17(1):204.

12. Hypertension in pregnancy. Report of the American College of Obstetricians and Gynecologists' Task Force on Hypertension in Pregnancy. Obstet Gynecol 2013, 122(5):1122-1131.

13. Gary Cunningham F KL, Steven Bloom,John Hauth,Dwight Rouse,Catherine Spong: Williams Obstetrics 23rd Edition. McGraw-Hill Professional 2009.

14. Stratta P, Canavese C, Dogliani M, Todros T, Gagliardi L, Vercellone A. Pregnancy-related acute renal failure. Clin Nephrol. 1989;32(1):14-20.

15. Prakash J, Pant P, Prakash S, Sivasankar M, Vohra R, Doley PK, Pandey LK, Singh U. Changing picture of acute kidney injury in pregnancy: Study of 259 cases over a period of 33 years. Indian J Nephrol. 2016;26(4):262-7.

16. Mahesh E, Puri S, Varma V, Madhyastha PR, Bande S, Gurudev KC. Pregnancy-related acute kidney injury: An analysis of 165 cases. Indian $J$ Nephrol. 2017;27(2):113-7.

17. Liang J, Li X, Kang C, Wang Y, Kulikoff XR, Coates MM, Ng M, Luo S, Mu Y, Wang $X$, et al. Maternal mortality ratios in 2852 Chinese counties, 19962015, and achievement of Millennium Development Goal 5 in China: a subnational analysis of the Global Burden of Disease Study 2016. Lancet. 2019;393(10168):241-52.

18. Li H, Liu K, Gu J, Zhang Y, Qiao Y, Sun X. The development and impact of primary health care in China from 1949 to 2015: A focused review. Int J Health Plann Manage. 2017:32(3):339-50.

19. Population Census Office under the State Council. Department of Population and Employment Statistics of National Bureau of Statistics. Tabulation on the 2010 population census of the People's Republic of China. Beijing: China Statistics Press 2012, http://www.stats.gov.cn/tjsj/pcsj/ rkpc/6rp/indexce.html.

20. Gao Y, Zhou H, Singh NS, Powell-Jackson T, Nash S, Yang M, Guo S, Fang H, Alvarez MM, Liu X, et al. Progress and challenges in maternal health in western China: a Countdown to 2015 national case study. Lancet Glob Health. 2017:5(5):e523-36.

21. Conti-Ramsden FI, Nathan HL, De Greeff A, Hall DR, Seed PT, Chappell LC, Shennan AH, Bramham K. Pregnancy-Related Acute Kidney Injury in Preeclampsia: Risk Factors and Renal Outcomes. Hypertension. 2019;74(5): 1144-51.

22. Drakeley AJ, Le Roux PA, Anthony J, Penny J. Acute renal failure complicating severe preeclampsia requiring admission to an obstetric intensive care unit. Am J Obstet Gynecol. 2002;186(2):253-6.

23. Ye W, Shu H, Yu Y, Li H, Chen L, Liu J, Li XM. Acute kidney injury in patients with HELLP syndrome. Int Urol Nephrol. 2019;51(7):1199-206.

24. Liu D, He W, Li Y, Xiong M, Wang L, Huang J, Jia L, Yuan S, Nie S. Epidemiology of acute kidney injury in hospitalized pregnant women in China. BMC Nephrol. 2019;20(1):67.

25. Gopalakrishnan N, Dhanapriya J, Muthukumar P, Sakthirajan R, Dineshkumar $\mathrm{T}$, Thirumurugan S, Balasubramaniyan T. Acute kidney injury in pregnancy-a single center experience. Ren Fail. 2015;37(9):1476-80.

26. Hildebrand AM, Liu K, Shariff SZ, Ray JG, Sontrop JM, Clark WF, Hladunewich MA, Garg AX. Characteristics and Outcomes of AKI Treated with Dialysis during Pregnancy and the Postpartum Period. J Am Soc Nephrol. 2015; 26(12):3085-91.

27. Liu Y, Ma X, Zheng J, Liu X, Yan T. Pregnancy outcomes in patients with acute kidney injury during pregnancy: a systematic review and metaanalysis. BMC Pregnancy Childbirth. 2017;17(1):235.
28. Crump C, Sundquist J, Winkleby MA, Sundquist K. Preterm birth and risk of chronic kidney disease from childhood into mid-adulthood: national cohort study. Bmj. 2019;365:11346.

29. Raju TNK, Pemberton VL, Saigal S, Blaisdell CJ, Moxey-Mims M, Buist S: Long-Term Healthcare Outcomes of Preterm Birth: An Executive Summary of a Conference Sponsored by the National Institutes of Health. J Pediatr 2017, 181:309-318.e301.

30. Nuyt AM, Lavoie JC, Mohamed I, Paquette K, Luu TM. Adult Consequences of Extremely Preterm Birth: Cardiovascular and Metabolic Diseases Risk Factors, Mechanisms, and Prevention Avenues. Clin Perinatol. 2017;44(2): 315-32.

31. Gonzales GF, Steenland K, Tapia V. Maternal hemoglobin level and fetal outcome at low and high altitudes. Am J Physiol Regul Integr Comp Physiol. 2009;297(5):R1477-85.

32. Jensen $G M$, Moore LG. The effect of high altitude and other risk factors on birthweight: independent or interactive effects? Am J Public Health. 1997; 87(6):1003-7.

33. Kametas NA, McAuliffe F, Krampl E, Chambers J, Nicolaides KH. Maternal cardiac function during pregnancy at high altitude. Bjog. 2004;111(10):1051-

34. Zamudio S, Palmer SK, Droma T, Stamm E, Coffin C, Moore LG. Effect of altitude on uterine artery blood flow during normal pregnancy. J Appl Physiol (1985). 1995;79(1):7-14

35. Wilson MJ, Lopez M, Vargas M, Julian C, Tellez W, Rodriguez A, Bigham A, Armaza JF, Niermeyer S, Shriver M, et al. Greater uterine artery blood flow during pregnancy in multigenerational (Andean) than shorter-term (European) high-altitude residents. Am J Physiol Regul Integr Comp Physiol. 2007;293(3):R1313-24.

36. Palmer SK, Moore LG, Young D, Cregger B, Berman JC, Zamudio S. Altered blood pressure course during normal pregnancy and increased preeclampsia at high altitude (3100 meters) in Colorado. Am J Obstet Gynecol. 1999;180(5):1161-8.

37. Bashir SO, Suekit H, Elkarib AO, Dafaalla MA, Abd Elrouf MB, Morsy MD, Eskandar M. The effect of high altitude on endothelial and vascular dysfunction markers in preeclamptic patients. Acta Physiol Hung. 2015; 102(4):391-9.

38. Hu XQ Dasqupta C, Xiao J, Yang S, Zhang L. Long-term high altitude hypoxia during gestation suppresses large conductance $\mathrm{Ca}(2+)$-activated $\mathrm{K}(+)$ channel function in uterine arteries: a causal role for microRNA-210. J Physiol. 2018:596(23):5891-906.

39. Kurlak LO, Mistry HD, Cindrova-Davies T, Burton GJ, Broughton Pipkin F. Human placental renin-angiotensin system in normotensive and preeclamptic pregnancies at high altitude and after acute hypoxiareoxygenation insult. J Physiol. 2016;594(5):1327-40.

40. Mistry HD, Kurlak LO, Broughton Pipkin F. The placental renin-angiotensin system and oxidative stress in pre-eclampsia. Placenta. 2013;34(2):182-6.

\section{Publisher's Note}

Springer Nature remains neutral with regard to jurisdictional claims in published maps and institutional affiliations.

Ready to submit your research? Choose BMC and benefit from:

- fast, convenient online submission

- thorough peer review by experienced researchers in your field

- rapid publication on acceptance

- support for research data, including large and complex data types

- gold Open Access which fosters wider collaboration and increased citations

- maximum visibility for your research: over $100 \mathrm{M}$ website views per year

At $\mathrm{BMC}$, research is always in progress.

Learn more biomedcentral.com/submissions 